\title{
Prevention of Catheter-Related Infections Using A Closed Hub System in Patients With Pulmonary Arterial Hypertension
}

\author{
Satoshi Akagi, MD; Hiromi Matsubara, MD; Aiko Ogawa, MD*; \\ Yusuke Kawai, MD; Kenichi Hisamatsu, MD; Katsumasa Miyaji, MD; \\ Mitsuru Munemasa, MD; Yoshihisa Fujimoto, MD; \\ Kengo Fukushima Kusano, MD*; Tohru Ohe, MD*
}

\begin{abstract}
Background Most of the patients with pulmonary arterial hypertension (PAH) receiving intravenous epoprostenol have experienced catheter-related infections during long-term treatment. Catheter hub was reported to be the most important source of catheter-related infections. To prevent the catheter-related infections, we have introduced a closed hub system and compared the incidence of catheter-related infections with that in patients using a non-closed hub system.

Methods and Results We evaluated the results obtained on 24 occasions in 20 patients with PAH between June 1999 and December 2005. On 11 occasions, a non-closed hub system was used and on 13 cases a closed hub system. We classified the catheter-related infection into a catheter-related bloodstream infection (CRBSI) group or a tunnel infection group based on the pathway of bacteria. The CRBSI rate was 0.89 per 1,000 catheter days in the non-closed hub system group vs 0.10 per 1,000 catheter days in the closed hub system group. Kaplan-Meier analysis showed that the risk of CRBSI significantly decreased in the closed hub system group. None of the patients died as a direct consequence of catheter-related infection during the study period.
\end{abstract}

Conclusions We successfully prevented CRBSI by using a closed hub system. (Circ J 2007; 71: 559-564)

Key Words: Catheter-related bloodstream infection; Catheter-related infection; Closed hub system; Epoprostenol; Pulmonary arterial hypertension

$\mathbf{P}$ ulmonary arterial hypertension $(\mathrm{PAH})$ is a rare disease of poor prognosis. Recently, continuous intravenous epoprostenol (EPO) was introduced as a treatment for PAH with the consequent improvement of the patients' quality of life! Although therapy with EPO improves the patients' exercise capacity and prognosis, various complications related to the central venous catheters arise during long-term treatment with EPO2,3 Among them, catheter-related infection is a serious problem, because the infections aggravate the primary disease.

The incidence of catheter-related infections was reported to be $0.3-9.1$ infections per 1,000 patient-days in patients with long-term, indwelling central venous catheters for various diseases $4-6$ According to a recent report by Oudiz et al, at least $10 \%$ of their patients with a catheter infection required admission to critical care wards and several patients died as a direct consequence of the catheter infection, although the incidence of catheter-related infections in patients with PAH receiving EPO was lower than that in patients with other diseases? Prevention of catheter-related infections would lead to further improvement of the prog-

(Received November 20, 2006; revised manuscript received December 25, 2006; accepted January 5, 2007)

Division of Cardiology, National Hospital Organization Okayama Medical Center, *Department of Cardiovascular Medicine Okayama University Graduate School of Medicine, Dentistry, and Pharmaceutical Sciences, Okayama, Japan

Mailing address: Satoshi Akagi, MD, Division of Cardiology, National Hospital Organization Okayama Medical Center, 1711-1 Tamasu, Okayama 701-1192, Japan. E-mail: sakagi-circ@umin.ac.jp nosis of patients with PAH.

It has already been reported that the catheter hub was the most important source of catheter-related infections ${ }^{8,9}$ and thus several closed hub systems have been introduced!10,11 In the present study, we adopted the closed hub system for patients with PAH receiving EPO to prevent bacterial invasion from the catheter hub.

\section{Methods}

\section{Closed Hub System}

We introduced the closed hub system after March 2002. This system consists of 2 parts (Fig 1). One part is the Hickman catheter connected to an extension tube (Figs 1A, $\mathrm{B}, \mathrm{D})$, and the other part is the infusion port for EPO in medication baggage (Fig 1C). The connection between the Hickman catheter and extension tube is a commercially available catheter connection system (I-system; Nipro Corporation, Japan). The I-system consists of an I-plug (a cap with a latex end injection plug with a male screw) and an I-set (a 21-gauge needle with a Luer-Lock female screw) (Fig 1B). The I-plug is applied to the hub of the Hickman catheter (Fig 1A). The needle of the I-set is inserted into the latex end of the I-plug. Then, the I-plug and I-set are fixed together by the Luer-Lock. The infusion port for EPO in the medication baggage consists of a plug made of latex (Fig 1C).

\section{Study Population}

From June 1999 to December 2005, 20 patients with 


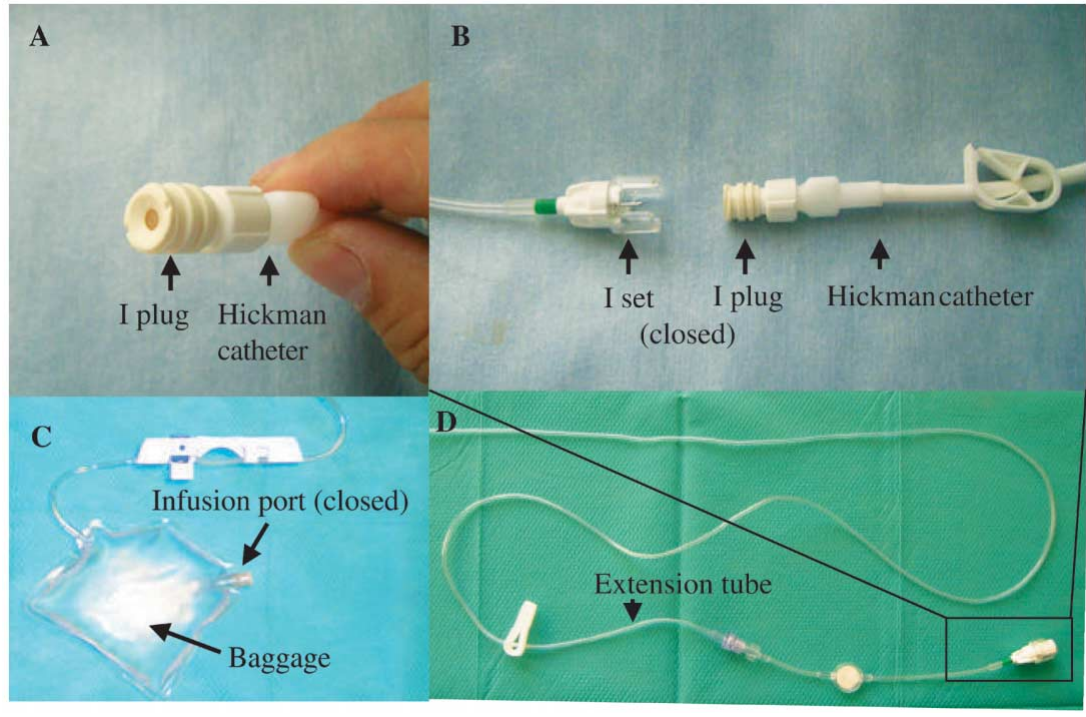

Fig 1. Closed hub system. (A) I-plug and Hickman catheter. (B) I-system consists of an I-plug and an I-set. (C) Infusion port for epoprostenol in medication baggage. (D) A whole picture of the closed hub system.
PAH receiving continuous therapy with EPO were referred to the National Hospital Organization Okayama Medical Center and to the Department of Cardiovascular Medicine of Okayama University. PAH was either idiopathic (19 patients) or caused by a connective tissue disease (1 patient) and was defined as PAH based on a mean pulmonary arterial pressure $\geq 25 \mathrm{mmHg}$ at rest, pulmonary capillary wedge pressure $\leq 15 \mathrm{mmHg}$ and a pulmonary vascular resistance of $\geq 240$ dyne $\cdot \mathrm{s} \cdot \mathrm{cm}^{-5}$. All patients underwent an infusion of EPO via a central venous catheter (Hickman; CR Bard, Inc, NJ, USA). The trained physicians inserted the catheters into the subclavian vein in the angiography room. Before the insertion, the skin was disinfected with $10 \%$ povidoneiodine or chlorhexidine gluconate. The physicians performed a thorough hand scrub, wore sterile gloves, gown, cap, and mask, and used large sterile drapes. In 11 patients (4 men and 7 women) who were started on continuous therapy with EPO before August 2002, we used the nonclosed hub system. And in 9 patients ( 2 men and 7 women) who were started on continuous therapy with EPO after January 2003, we used the closed hub system. In addition, in 4 patients (all women) who had suffered catheter-related infections with the non-closed hub system, this was changed to the closed hub system after March 2003. Patients were discharged from hospital after they were instructed on the care and maintenance of the catheter. All patients were monitored every 1-4 months. During the intervals, their private physicians were in the charge of the catheter care and maintenance. All patients were not treated with oral steroids. And there were no patients with immunodeficiency. The local institutional review boards approved the protocol of this study. All participating patients provided informed consent to participate in the study.

\section{Care and Maintenance of the Catheter}

The administration of EPO required a daily mixing of the drug in both systems. At first in the non-closed hub system, patients had to fill a backup infusion pump reservoir with EPO under sterile conditions. Then, the patients had to disconnect the catheter from the infusing pump and quickly connect it to the newly filled pump. Thus, the patients' extension tube connected to their central venous catheter, which was 'opened' every day.
In the closed hub system, the EPO was refilled from the infusion port in the medication baggage under sterile conditions. All the patients had to change the medication baggage and the extension tube every 3 days. Because the central venous catheter was closed by the I-plug, the catheter was never 'opened' while they were at home. A physician changed the I-plug using a sterile procedure when the patient visited the outpatient clinic every month. The tube and central venous catheter was reconnected using sterile procedures, without the use of a saline solution or heparin.

A sterile dry gauze or semi-permeable dressing was used to seal the catheter insertion site under sterile conditions every day. The skin around the catheter insertion site was disinfected with povidone-iodine or chlorhexidine gluconate at the time the dressing was changed. This dressing regimen was not changed over time. Physicians and nurses were instructed on the care and maintenance of the patient catheters. And patients were instructed to ask a nurse and/or a physician for an evaluation immediately if they noted erythema, edema, tenderness and/or a persistent discharge at the catheter insertion site or generalized low-grade fever.

\section{Definitions of Catheter-Related Infections}

When the patients suspected a catheter-related infection, they were instructed to immediately visit our hospital or a private physician. When the private physician diagnosed a catheter-related infection, he/she must have referred the patient to our hospital for admission or prescribed antibiotics. In fact, patients were admitted to our hospital when catheter-related infections did not improve after physician treatment. At the hospital, a chest radiograph was taken, and blood and urine examinations were carried out. We diagnosed a patient as having a catheter-related infection according to the $2002 \mathrm{CDC}$ recommendations!2 Catheterrelated bloodstream infections (CRBSI) was defined as at least 1 positive blood culture obtained from a peripheral vein, in a patient with clinical manifestations of infections (ie, fever, chills, and/or hypotension) and if there was no apparent source for bloodstream infection except for the catheter. Tunnel infection was defined as erythema, edema, tenderness, or drainage from the exit site. If necessary, we removed the Hickman catheter and cultured the catheter tip and pus from the insertion site. 
Table 1 Patient Demographics

\begin{tabular}{lccc}
\hline \hline Variables & $\begin{array}{c}\text { All patients } \\
(n=20)\end{array}$ & $\begin{array}{c}\text { Non-closed hub system } \\
(n=11)\end{array}$ & $\begin{array}{c}\text { Closed hub system } \\
(n=13)\end{array}$ \\
\hline Age, years & $31.3 \pm 11.3$ & $26.3 \pm 7.2$ & $35.5 \pm 11.0$ \\
M/F & $6 / 20$ & $4 / 11$ & $2 / 13$ \\
Diagnoses & & & 12 \\
$\quad$ Idiopathic PAH & 20 & 11 & 1 \\
$\quad$ CTD & 0 & 0 & 8,838 \\
Catheter days & 14,732 & 5,894 & \\
\hline
\end{tabular}

$P A H$, pulmonary arterial hypertension; CTD, connective tissue disease.

Table 2 Number of Catheter Infections in Patients With PAH Distributed According to the Type of System Used

\begin{tabular}{|c|c|c|c|c|c|c|}
\hline Patient no. & Sex & Age (years) & System & $\begin{array}{l}\text { Incidence of all } \\
\text { catheter infection }\end{array}$ & $\begin{array}{l}\text { Incidence of } \\
\text { tunnel infection }\end{array}$ & $\begin{array}{c}\text { Incidence of } \\
\text { CRBSI }\end{array}$ \\
\hline 1 & $F$ & 23 & Non-closed & 1 & 0 & 1 \\
\hline 2 & $F$ & 33 & Non-closed & 3 & 1 & 2 \\
\hline 3 & $M$ & 20 & Non-closed & 3 & 2 & 1 \\
\hline 4 & $M$ & 21 & Non-closed & 3 & 2 & 1 \\
\hline 5 & $F$ & 26 & Non-closed & 5 & 5 & 0 \\
\hline 6 & $F$ & 36 & Non-closed & 1 & 1 & 0 \\
\hline 7 & $M$ & 14 & Non-closed & 2 & 1 & 1 \\
\hline 8 & $M$ & 19 & Non-closed & 2 & 1 & 1 \\
\hline 9 & $F$ & 28 & Non-closed & 1 & 1 & 0 \\
\hline 10 & $F$ & 31 & Non-closed & 0 & 0 & 0 \\
\hline 11 & $F$ & 36 & Non-closed & 0 & 0 & 0 \\
\hline 12 & $F$ & 51 & Closed & 2 & 0 & 2 \\
\hline 13 & $F$ & 32 & Closed & 1 & 1 & 0 \\
\hline 14 & $M$ & 58 & Closed & 0 & 0 & 0 \\
\hline 15 & $F$ & 35 & Closed & 0 & 0 & 0 \\
\hline 16 & $F$ & 33 & Closed & 0 & 0 & 0 \\
\hline 17 & $F$ & 29 & Closed & 1 & 1 & 0 \\
\hline 18 & $M$ & 32 & Closed & 0 & 0 & 0 \\
\hline 19 & $F$ & 16 & Closed & 0 & 0 & 0 \\
\hline 20 & $F$ & 45 & Closed & 0 & 0 & 0 \\
\hline $2 *$ & $F$ & 33 & Closed & 0 & 0 & 0 \\
\hline $5 *$ & $F$ & 26 & Closed & 1 & 1 & 0 \\
\hline $6 *$ & $F$ & 36 & Closed & 0 & 0 & 0 \\
\hline $9 *$ & $F$ & 28 & Closed & 1 & 1 & 0 \\
\hline
\end{tabular}

*Patients who were switched from the non-closed hub system.

CRBSI, catheter-related bloodstream infection. Other abbreviation see in Table 1.

Table 3 Bacterial Species Isolated From Blood, Catheter Tip or Pus From the Insertion Site of PAH Patients

\begin{tabular}{|c|c|c|c|c|}
\hline \multirow{2}{*}{ Organism } & \multicolumn{2}{|c|}{ Instances of a non-closed hub system } & \multicolumn{2}{|c|}{ Instances of a closed hub system } \\
\hline & Tunnel infection & CRBSI & Tunnel infection & CRBSI \\
\hline Methicillin-sensitive staphylococcus aureus & 5 & 2 & 2 & 0 \\
\hline Micrococcus spp & 1 & 1 & 0 & 1 \\
\hline Staphylococcus epidermidis & 0 & 1 & 0 & 0 \\
\hline Coagulase negative staphylococci & 1 & 0 & 0 & 0 \\
\hline Corynebacterium spp & 0 & 0 & 1 & 0 \\
\hline Not cultured & 4 & 3 & 1 & 1 \\
\hline Not collected & 3 & 0 & 0 & 0 \\
\hline
\end{tabular}

Figs indicate the number of times the microorganisms were isolated.

Abbreviations see in Tables 1,2.

\section{Statistical Analysis}

Results are reported as the mean \pm standard deviation. Infection rates are reported as per 1,000 patient days. The cumulative risk of developing a catheter-related infection in the 2 groups as a function of the duration of catheterization was estimated according to the Kaplan-Meier method and compared with the use of the log rank test.

\section{Results}

The patient characteristics are shown in Table 1. The patients' age ranged from 15 to 58 years (mean age 31.3 years). The total number of catheter days was 5,894 days in the non-closed hub system group and 8,838 days in the closed hub system group. The duration of catheter indwelling was 281-1306 days, with an average of 536 \pm 367 days in the non-closed hub system group and 50-1,386 days, with an average of $680 \pm 370$ days in the closed hub system group.

In the non-closed hub system group, 9 patients developed a total of 21 catheter-related infections during the study period (Table 2). Eight patients (14 catheter infections) had 


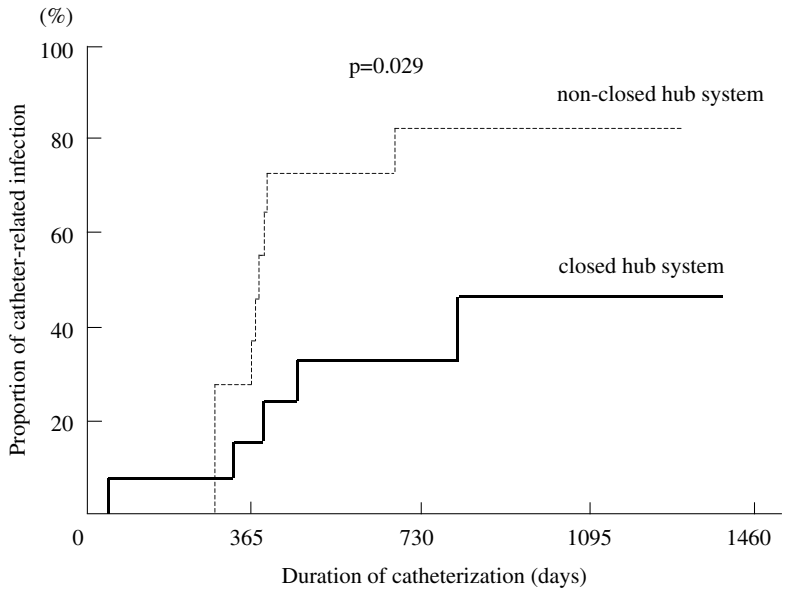

Fig 2. Analysis of the Kaplan-Meier estimates of the overall risk for catheter-related infections according to the duration of the catheterization.

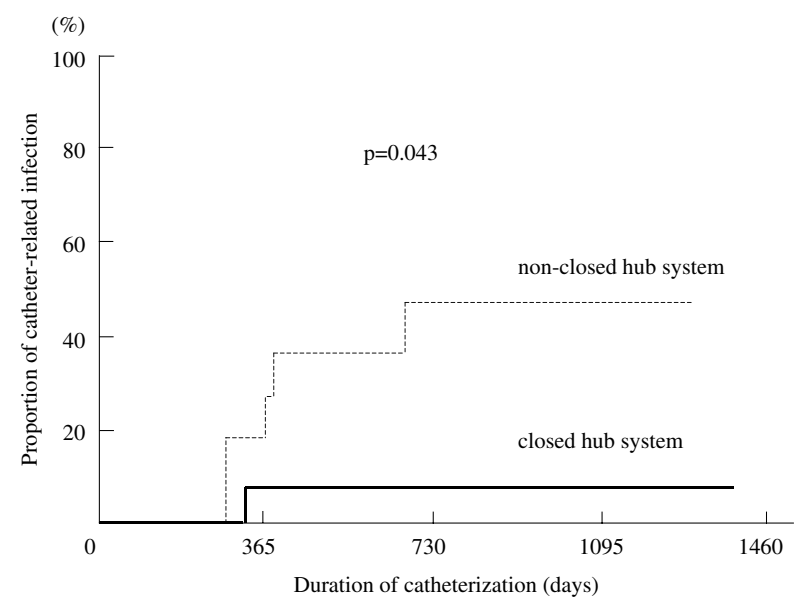

Fig 3. Analysis of the Kaplan-Meier estimates of the risk for catheter-related bloodstream infection according to the duration of the catheterization.

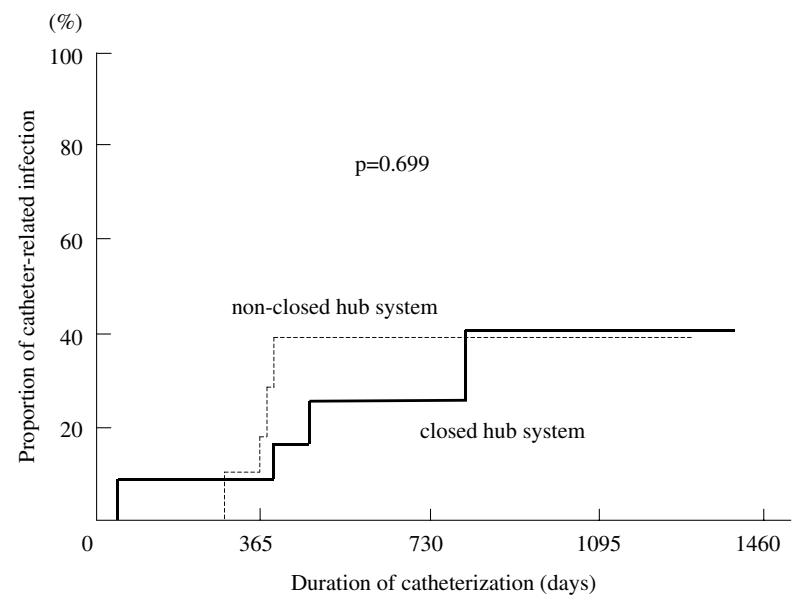

Fig 4. Analysis of the Kaplan-Meier estimates of the risk for tunnel infections according to the duration of the catheterization. tunnel infections and 6 patients (7 catheter infections) had CRBSI. Five patients developed both types of infections.

In the closed hub system group, 5 patients developed a total of 6 catheter-related infections during the study period (Table 2). Four patients (4 catheter infections) had tunnel infections and 1 ( 2 catheter infections) had CRBSI. Two of the patients who developed tunnel infections had previously used the non-closed hub system, and one of them had suffered repeated tunnel infections (5 catheter infections) with the non-closed hub system.

All patients were admitted to either of our 2 centers. In the patients with tunnel infection, the catheter had to be removed as part of the treatment and they were administered antibiotics intravenously at least once a week, except for 1 patient. In 1 patient, treatment with just oral antibiotics was sufficient to eradicate the infection.

The etiologic organism was identified in at least one cultured specimen in 13 of the 27 clinical episodes of infections. The organisms isolated are listed in Table 3. Methicillin-sensitive staphylococcus aureus (MSSA) was the most frequent isolate in both groups. In the non-closed hub system, bacterial cultures were negative in 7 of the 21 clinical infections.

The overall catheter-related infection rate for our patients was 1.53 per 1,000 catheter days in the non-closed hub system group vs 0.45 per 1,000 catheter days in the closed hub system group. The CRBSI rate was 0.89 per 1,000 catheter days in the non-closed hub system group vs 0.10 per 1,000 catheter days in the closed hub system group. The tunnel infection rate was 0.87 per 1,000 catheter days in the non-closed hub system group vs 0.33 per 1,000 catheter days in the closed hub system group.

Kaplan-Meier estimates of the overall risk for catheterrelated infections showed a significant difference between the non-closed hub system and the closed hub system (Fig 2, p=0.029). Furthermore, Kaplan-Meier estimates of the risk for CRBSI showed a significant difference between the non-closed hub system and the closed hub system (Fig 3, p=0.043). However, Kaplan-Meier estimates of the risk for tunnel infections showed no difference between the 2 groups (Fig 4, p=0.699).

None of the patients died as a direct consequence of a catheter-related infection during the study period.

\section{Discussion}

In the present study, the overall incidence of catheterrelated infections and CRBSI was significantly decreased with the closed hub system. Furthermore, the incidence of CRBSI was extremely low in comparison to that found in a previous study?

Recently, the use of continuous infusion therapies at home, for example, total parenteral nutrition, has remarkably increased. This type of therapy has contributed not only to an increase in therapeutic options but also in terms of improving patients' quality of life. However, various problems associated with the management of this therapy still persist. Catheter-related infections, one of the most serious problems, can aggravate the primary disease and worse, might result in death. Catheter-related infections associated with the therapy have been evaluated with regard to various diseases ${ }^{13-16}$

In the present study, catheter-related infections were divided into 2 groups according to the 2002 CDC recommendations. This difference can be explained by the path- 
ways of bacterial invasion. CRBSI could be caused by bacterial invasion through the catheter connection. Tunnel infection could be caused by direct bacterial invasion through the catheter insertion site. To prevent catheterrelated infections, we need to identify which pathway of bacterial invasion was predominant in an individual case.

Previously, it was considered that direct bacterial invasion through the catheter insertion site was the main cause of catheter-related infections ${ }^{17,18}$ To prevent direct bacterial invasion, it was thought that the catheter insertion site had to be cleaned and the subcutaneous tunnel had to be long. However, it was reported that the results of bacterial culture from the catheter insertion site did not necessarily coincide with those of blood and catheter tip cultures ${ }^{19,20}$ In addition, the use of long subcutaneous tunnels failed to decrease the frequency of catheter-related infections ${ }^{21,22}$ As a result, attention has now focused on bacterial invasion through the catheter connection. Sitges-Serra et al evaluated the possibility of bacterial contamination at the catheter connection and postulated the "hub hypothesis". They reported that the hub of a catheter was the most important source of catheter-related infections? This hypothesis has been supported by several studies.23-26 Consequently, we adopted the closed hub system for the catheter connection in our patients. The system we used simplifies catheter care and infusion line use compared with the non-closed hub system. Bacterial contamination can be minimized with this system because the catheter connection is not open to air during the change of the infusion line except at the monthly change of the I-plug in the clinic. The effectiveness of this system in reducing CRBSI was already reported in patients with other diseases 10 In the present study, we showed that we could significantly decrease CRBSI by using the closed hub system in patients with $\mathrm{PAH}$ receiving EPO.

As for microorganisms causing catheter infections, we found that MSSA was the most frequent cause of infection, which is in agreement with the results of a previous study involving patients with $\mathrm{PAH}$ ? In that study, micrococcus spp was the second most common etiologic agent. The clinical syndrome of micrococcal catheter infection presented as generalized weakness and fatigue with or without fever? Although this symptom was common in the case of CRBSI with a non-closed hub system, cultures were positive in 1 patient. This could be because of micrococcus spp might have been present; this was ignored as a possible cause in our institute until 2003 ? $^{7}$

Although we could decrease the overall occurrence of catheter-related infections by reducing CRBSI, approximately $50 \%$ of patients still experienced catheter-related infections during 3 years. Most of the catheter-related infections were of the tunnel type and thus it is important to focus on the prevention of tunnel infections in the future.

When a patient develops a catheter-related infection, the clinician must treat it as early as possible. However, early detection of catheter-related infections is difficult. Thus, when a patient receiving EPO starts to complain of constitutional symptoms for no apparent reason, clinicians should suspect the possibility of catheter-related infections and carry out a thorough investigation to rule out this possibility.

This study has several limitations. This is an observational and not a prospective study. We might have underestimated the true incidence of catheter-related infections. If the catheter infection was slight, clinicians might have been unaware of it. Clinicians treat catheter-related infections with antibiotics based on the diagnosis of other infections.

\section{Conclusions}

The present study demonstrated that the use of the closed hub system reduced the overall occurrence of catheterrelated infections in patients with $\mathrm{PAH}$ receiving continuous therapy with EPO at home. Furthermore, the risk of CRBSI was significantly decreased with this system. Besides, the incidence of CRBSI was low compared with another study involving patients with $\mathrm{PAH}$ receiving continuous therapy with EPO.

\section{References}

1. McLaughlin VV, Shillington A, Rich S. Survival in primary pulmonary hypertension: The impact of epoprostenol therapy. Circulation 2002; 106: $1477-1482$.

2. Ogawa A, Matsubara H, Fujio H, Miyaji K, Nakamura K, Morita H, et al. Risk of alveolar hemorrhage in patients with primary pulmonary hypertension. Circ J 2005; 69: 216-220.

3. Kataoka M, Satoh T, Manabe T, Anzai T, Yoshikawa T, Mitamura $\mathrm{H}$, et al. Oral sildenafil improves primary pulmonary hypertension refractory to epoprostenol. Circ J 2005; 69: 461-465.

4. van Hoff J, Berg AT, Seashore JH. The effect of right atrial catheters on infectious complications of chemotherapy in children. J Clin Oncol 1990; 8: 1255-1262.

5. Decker MD, Edwards KM. Central venous catheter infections. Pediatr Clin North Am 1988; 35: 579-612.

6. Moureau N, Poole S, Murdock MA, Gray SM, Semba CP. Central venous catheters in home infusion care: Outcomes analysis in 50,470 patients. J Vasc Interv Radiol 2002; 13: 1009-1016.

7. Oudiz RJ, Widlitz A, Beckmann XJ, Camanga D, Alfie J, Brundage $\mathrm{BH}$, et al. Micrococcus-associated central venous catheter infection in patients with pulmonary arterial hypertension. Chest 2004; 126: 90-94.

8. Sitges-Serra A, Linares J, Garau J. Catheter sepsis: The clue is the hub. Surgery 1985; 97: 355-357.

9. Sitges-Serra A, Puig P, Linares J, Perez JL, Farrero N, Jaurrieta E, et al. Hub colonization as the initial step in an outbreak of catheter-related sepsis due to coagulase negative staphylococci during parenteral nutrition. JPEN J Parenter Enteral Nutr 1984; 8: 668-672.

10. Inoue Y, Nezu R, Matsuda H, Fujii M, Nakai S, Wasa M, et al. Prevention of catheter-related sepsis during parenteral nutrition: Effect of a new connection device. JPEN J Parenter Enteral Nutr 1992; 16: $581-585$.

11. Bouza E, Munoz P, Lopez-Rodriguez J, Jesus Perez M, Rincon C, Martin Rabadan P, et al. A needleless closed system device (CLAVE) protects from intravascular catheter tip and hub colonization: A prospective randomized study. J Hosp Infect 2003; 54: 279 287.

12. O'Grady NP, Alexander M, Dellinger EP, Gerberding JL, Heard SO, Maki DG, et al. Guidelines for the prevention of intravascular catheter-related infections. MMWR Recomm Rep 2002; 51: 1-29.

13. Ryan JA Jr, Abel RM, Abbott WM, Hopkins CC, Chesney TM, Colley R, et al. Catheter complications in total parenteral nutrition: A prospective study of 200 consecutive patients. N Engl J Med 1974; 290: $757-761$.

14. Goldmann DA, Martin WT, Worthington JW. Growth of bacteria and fungi in total parenteral nutrition solutions. Am J Surg 1973; 126: $314-318$.

15. Sanders RA, Sheldon GF. Septic complications of total parenteral nutrition: A five year experience. Am J Surg 1976; 132: 214-220.

16. Snydman DR, Murray SA, Kornfeld SJ, Majka JA, Ellis CA. Total parenteral nutrition-related infections: Prospective epidemiologic study using semiquantitative methods Am J Med 1982; 73: 695-699.

17. Maki DG, Weise CE, Sarafin HW. A semiquantitative culture method for identifying intravenous-catheter-related infection. $N$ Engl J Med 1977; 296: $1305-1309$.

18. Snydman DR, Gorbea HF, Pober BR, Majka JA, Murray SA, Perry LK. Predictive value of surveillance skin cultures in total parenteral nutrition related infection. Lancet 1982; 2: 1385-1388.

19. Bjornson HS, Colley R, Bower RH, Duty VP, Schwartz-Fulton JT, Fischer JE. Association between microorganism growth at the catheter insertion site and colonization of the catheter in patients receiving total parenteral nutrition. Surgery 1982; 92: 720-727. 
20. Jarrard MM, Freeman JB. The effects of antibiotic ointments and antiseptics on the skin flora beneath subclavian catheter dressings during intravenous hyperalimentation. J Surg Res 1977; 22: $520-$ 526.

21. Sitges-Serra A, Puig P, Jaurrieta E, Garau J, Alastrue A, Sitges-Creus A. Catheter sepsis due to staphylococcus epidermidis during parenteral nutrition. Surg Gynecol Obstet 1980; 151: 481-483.

22. Von Meyenfeldt MM, Stapert J, De Jong PC, Soeters PB, Wesdorp RI, Greep JM. TPN catheter sepsis: Lack of effect of subcutaneous tunneling of PVC catheters on sepsis rate. JPEN J Parenter Enteral Nutr 1980; 4: 514-517.

23. Holm I, Wretlind A. Prophylaxis against infection and septicemia in parenteral nutrition via central venous catheter. Acta Chir Scand
1975; 141: 173-181.

24. Deitel M, Krajden S, Saldanha CF, Gregory WD, Fuksa M, Cantwell E. An outbreak of Staphylococcus epidermidis septicemia. JPEN J Parenter Enteral Nutr 1983; 7: 569-572.

25. Forse RA, Dixon C, Bernard K, Martinez L, McLean AP, Meakins JL. Staphylococcus epidermidis: An important pathogen. Surgery 1979; 86: 507-514.

26. Stotter AT, Ward H, Waterfield AH, Hilton J, Sim AJ. Junctional care: The key to prevention of catheter sepsis in intravenous feeding. JPEN J Parenter Enteral Nutr 1987; 11: 159-162.

27. Yap RL, Mermel LA. Micrococcus infection in patients receiving epoprostenol by continuous infusion. Eur J Clin Microbiol Infect Dis 2003; 22: 704-705. 